\title{
RABDAM: quantifying specific radiation damage in individual protein crystal structures
}

Kathryn L. Shelley*, Thomas P. E. Dixon, Jonathan C. Brooks-Bartlett and Elspeth F. Garman*

Department of Biochemistry, University of Oxford, South Parks Road, Oxford, OX1 3QU, United Kingdom.

*Correspondence e-mails: kathryn.1.shelley@gmail.com, elspeth.garman@ bioch.ox.ac.uk

\section{Supporting information}
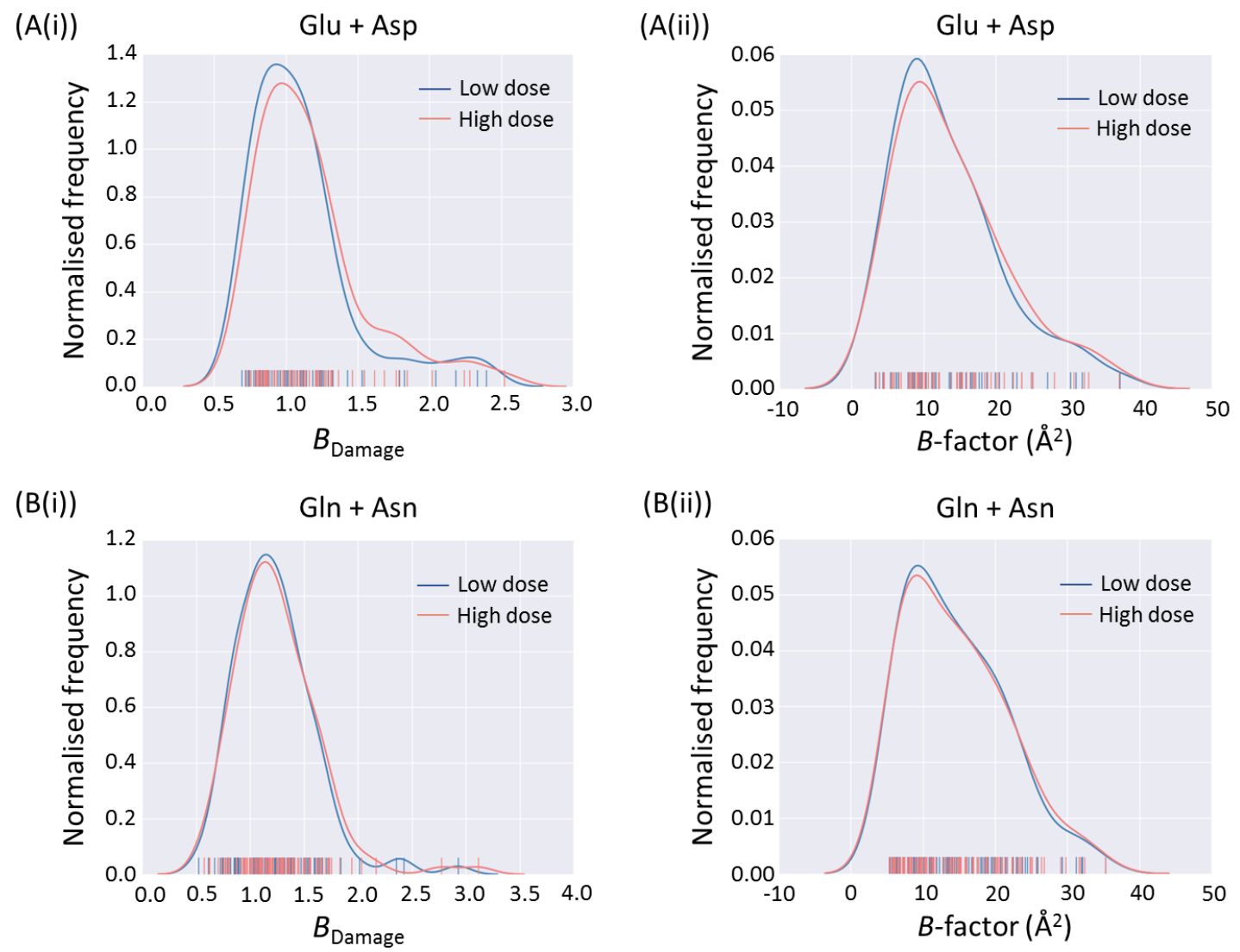

Figure S1: $B_{\text {Damage }}$ highlights expected sites of specific radiation damage, which are not readily detectable from $B$-factor alone, in PX structures. (A) There is a larger difference between the (i) $B_{\text {Damage }}$

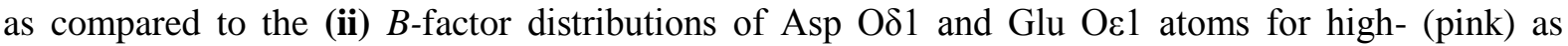
compared to low- (blue) dose datasets. (B) In contrast, there is little discernible difference between both the (i) $B_{\text {Damage }}$ and (ii) $B$-factor distributions of Asn $\mathrm{O} \delta 1$ and Gln $O \varepsilon 1$ atoms (which are not expected to be sites of specific radiation damage induced chemical changes). The low- and high-dose distributions relate respectively to the low- and high-dose datasets collected from each of the six proteins (elastase, ribonuclease A, thaumatin, trypsin, lysozyme and insulin) studied in Nanao et al. (2005). Plots are smoothed via a kernel density estimator; $B_{\text {Damage }}$ values of individual atoms are represented as rug plots, and were calculated using default program parameter values (i.e. a packing density radius of $7 \AA$ and a sliding window size of $2 \%$ - see section $\$ 2.1$ and the online program manual). 


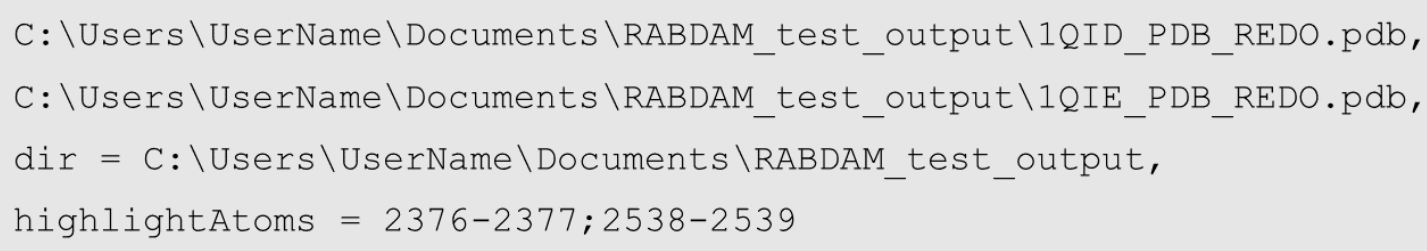

Figure S2: The RABDAM input file for the $B_{\text {Damage }}$ analysis, presented in Figure 4 and Table 3, of the first two datasets in the Torpedo californica acetylcholinesterase radiation damage series collected by Weik et al. (2000). Because the original structures from this series deposited in the PDB were not subjected to per-atom $B$-factor refinement, as is a requirement for $B_{\text {Damage }}$ calculation, the analysis was instead performed upon the updated, per-atom refined structures downloaded from the PDB_REDO databank; hence the input structures are specified by local PDB file paths as opposed to PDB accession codes. The highlightAtoms parameter is used to highlight the $B_{\text {Damage }}$ values of the O $\varepsilon$ atoms of Glu36 and Glu327 on the output kernel density estimate. 